OPEN ACCESS

Edited by:

Marco Sarà,

San Raffaele Cassino, Italy

Reviewed by:

Sergio Bagnato,

Fondazione Istituto

San Raffaele G. Giglio, Italy

Anna Estraneo,

Istituti Clinici Scientifici

Maugeri Spa SB, Italy

*Correspondence:

Xinhuai Wu

wuxinhuai_beijing@163.com

tThese authors have contributed equally to this work.

Specialty section:

This article was submitted to Neurotrauma,

a section of the journal

Frontiers in Neurology

Received: 07 November 2017 Accepted: 14 February 2018

Published: 02 March 2018

Citation:

Wu B, Yang Y, Zhou S, Wang W, Wang Z, Hu G, He J and Wu X (2018) Could Arterial Spin Labeling Distinguish Patients in Minimally Conscious State from Patients in Vegetative State? Front. Neurol. 9:110. doi: 10.3389/fneur.2018.00110

\section{Could Arterial Spin Labeling Distinguish Patients in Minimally Conscious State from Patients in Vegetative State?}

\author{
Bing $W u^{1+}$, Yi Yang ${ }^{2 \dagger}$, Shuai Zhou ${ }^{3}$, Wei Wang ${ }^{1}$, Zizhen Wang ${ }^{1}$, Gang $H^{1}$, Jianghong $\mathrm{He}^{2}$ \\ and Xinhuai $W^{1,3 *}$

\begin{abstract}
'Department of Radiology, PLA Army General Hospital, Beijing, China, ${ }^{2}$ Department of Neurosurgery, PLA Army General
\end{abstract} \\ Hospital, Beijing, China, ${ }^{3}$ Inner Mongolia Medical University, Hohhot, China
}

Purpose: Diagnostic error is common among patients with vegetative state (VS) and minimally conscious state (MCS). The purpose of this article is to use three-dimensional pseudo-continuous arterial spin labeling (pcASL) to compare cerebral blood flow (CBF) patterns in patients in MCS with those in VS.

Methods: Patients meeting MCS and VS criteria were identified. Two post-labeling delay (PLD) time pcASL on 3.0-Tesla magnetic resonance imaging scanner system were performed with patients in the resting awake state. After registration to T1WI structure imaging, multiple brain regions of interest of ASL CBF map were automatically separated. The average CBF value of every brain region was calculated and compared between the MCS and VS groups with $t$-tests.

Results: Fifteen patients with VS were identified, with ages ranging from 33 to 71 years. Eight patients who met the MCS criteria ranged in age from 23 to 61 years. Compared with VS, the regional CBF for MCS had a pattern of significantly increased CBF in the regions including the putamen, anterior cingulate gyrus, and medial frontal cortex. A left-lateralized pattern was observed to differentiate MCS from VS. CBF with PLD $2.5 \mathrm{~s}$ could find more regions of pattern differentiating MCS from VS than with PLD $1.5 \mathrm{~s}$, except for the pallidum.

Conclusion: MCS might be differentiated from VS by different ranges of regional CBF as measured by ASL. Multi-PLD ASL may serve as an adjunct method to separate MCS from VS and assess functional reserve in patients recovering from severe brain injuries.

Keywords: disorders of consciousness, minimally conscious state, arterial spin labeling

\section{INTRODUCTION}

A subset of coma patients develops a prolonged impairment in consciousness, such as the vegetative state (VS) and minimally conscious state (MCS). Diagnostic error is common among patients with VS and MCS (1). Because of variable behavior observed at the bedside, approximately $30-40 \%$ of people diagnosed with VS actually retain conscious awareness (2).

Abbreviations: ASL, arterial spin labeling; CBF, cerebral blood flow; CRS-R, Coma Recovery Score--Revised; DMN, default mode network; GM, gray matter; MCS, minimally conscious state; VS, vegetative state; WM, white matter. 
Arterial spin labeling (ASL) is a magnetic resonance perfusion method that measures cerebral blood flow (CBF) in vivo. Unlike other comparable functional imaging modalities, ASL avoids the use of a radioactive tracer or gadolinium and is noninvasive. Recent advances in magnetic resonance imaging (MRI) technology, including higher magnetic fields, array receiver coils, pseudo-continuous arterial spin labeling (pcASL) sequences, and rapid three-dimensional (3D) acquisition techniques, have made it feasible to apply ASL in a wide range of clinical applications (3-9), especially in the characterization of brain states, longitudinal follow-up, or monitoring treatment effects.

Cerebral blood flow has been demonstrated to have a strong association with neural activity (10). Preliminary studies suggest a marked reductions in cerebral metabolism in MCS patients (11), suggesting that CBF may also be decreased.

A previous study identified globally decreased $\mathrm{CBF}$ and a selective reduction of $\mathrm{CBF}$ within the frontal cortical regions as well as gray matter (GM) in MCS patients compared with normal control subjects (12). The purpose of our study was to use 3D pcASL to compare CBF patterns in MCS patients with those in VS patients.

\section{MATERIALS AND METHODS}

\section{Patient Selection}

The study was approved by the Ethics Committee of the PLA Army General Hospital. Consent for this study was obtained for patients from a legally authorized representative. Control subjects provided their own consent. Patients who met the criteria for MCS and VS were identified through discussions with physicians in the inpatient Neurosurgery Department of the PLA Army General Hospital between 2012 and 2016. Clinical subjects were adults who had sustained stroke, traumatic brain injury, or hypoxic ischemic injury. Subjects were included if they were between 18 and 75 years of age, had non-progressive severe brain injury, were at least 1 month post-injury, and met the Aspen Consensus Conference criteria for MCS (13) and VS. Subjects were excluded if they were ventilator-dependent, had a refractory seizure disorder, or had an MRI-incompatible device. All subjects received a neurologic examination, including a Coma Recovery Scale--Revised (14) assessment, on initial evaluation. The clinical evaluation was made at least four times in 2 weeks before the ASL study, and the last assessment was done 3 to 7 days before the ASL study. The diagnosis of VS and MCS was made when the last three assessments were equal. All neurologic examinations were done by $\mathrm{YY}$ and $\mathrm{HJ}$, who had 10 and 18 years of experience as neurosurgeons, respectively. Healthy control subjects, aged from 20 to 70 years, were recruited by advertisement. Study exclusion criteria for healthy volunteers included contraindications to MRI; pregnancy, major head trauma, abnormal structural MRI, and the presence of other neurological diseases. Excluded medications included psychoactive medications, nitrates, and warfarin or other drugs that may affect CBF.

\section{MRI Protocol}

Patients and control subjects underwent structural and functional imaging studies. All image data were acquired on a 3.0-Tesla MRI scanner system (GE Medical Systems, Milwaukee, WI, USA). Structure imaging included 3D-T1-weighted and T2-weighted images. ASL sequences were obtained during the awake resting state. Patient and control ASL sequences with significant motion degradation were excluded from analysis.

The raw ASL images were acquired twice using 3D pcASL sequences with a post-labeled delay (PLD) time of 1.5 or $2.5 \mathrm{~s}$ (15-17). Images were acquired with the following parameters: 512 sampling points on eight spirals, spatial resolution $=3.64 \mathrm{~mm}$, $\mathrm{TR}=4,590(\mathrm{PLD}=1.5 \mathrm{~s}) / 5,285 \mathrm{~ms}(\mathrm{PLD}=2.5 \mathrm{~s}), \mathrm{TE}=10.5 \mathrm{~ms}$, slice thickness $=4 \mathrm{~mm}$, number of slices $=36$, acquisition time $=4: 29(\mathrm{PLD}=1.5 \mathrm{~s}) / 5: 09(\mathrm{PLD}=2.5 \mathrm{~s})$ minutes, field of view $(\mathrm{FOV})=24 \mathrm{~cm}$, and number of excitations $(\mathrm{NEX})=3$. A high-resolution volumetric T1-weighted sequence of the whole brain were acquired with the following parameters: $\mathrm{TR}=8.2 \mathrm{~ms}$, $\mathrm{TE}=3.2 \mathrm{~ms}$, TI $=450 \mathrm{~ms}, \mathrm{FOV}=24 \mathrm{~cm}$, slice thickness $=1$ $\mathrm{mm}$, number of slices $=156$, acquisition time $=4: 08 \mathrm{~min}$, matrix $=256 \times 256$, and NEX $=1$. The CBF map was calculated with commercial software on the GE AW workstation. The psASL has been demonstrated to be both precise and reliable compared with the gold standard 15O-water PET (18).

\section{Analysis Methods}

The CBF maps were registered to 3D-T1WI structure imaging, and the 3D-T1WI images were used for image registration and normalization into a standardized space (Montreal Neurological Institute template, MNI space) within the Statistical Parametric Mapping (SPM8) ${ }^{1}$ on MatLab 7 (MathWorks, Natick, MA, USA). Anatomical regions-of-interest (ROIs) were generated from WFU Pickatlas (Wake Forest University). ${ }^{2}$ Ten typical combined bilateral ROIs were selected on the basis of a literature review of previous ASL studies and included the caudate, putamen (PUT), thalamus (THAL), anterior cingulate gyrus, medial frontal cortex, middle frontal cortex, superior temporal gyrus, posterior cingulate, parietal cortex, and occipital pole (19). Next, multiple brain ROIs of ASL CBF map were automatically separated based on the Automated Anatomical Labeling-116 (AAL-116) brain template (20) and the bilateral Brodmann's template, as AAL and Brodmann's templates are generally used in previous function imaging studies. On the AAL temple, we choose ROIs on left and right separately. We decided to omit cerebellar values from our analysis, given that imaging coverage of the cerebellum using the pulsed-continuous method is variable and subject to systematic error (21).

The average $\mathrm{CBF}$ was calculated for each structure for both control subjects and patients. Then the average CBF value of every brain region was compared between the MCS and VS groups with $t$-tests.

For a global analysis of CBF, GM was separated within the WFU Pickatlas template. Two deep white matter (WM) masks were manually drawn to avoid GM-WM contamination $(18,22)$. This was done by selecting sphere-shaped voxels with a $10-\mathrm{mm}$ radius at the right and left centrum ovale.

${ }^{1}$ http://www.fil.ion.ucl.ac.uk/spm/software/spm8/.

${ }^{2}$ http://fmri.wfubmc.edu/cms/software. 
Differences in CBF in GM and WM between patients and controls were calculated using a two-sample $t$-test assuming unequal variances.

\section{RESULTS}

\section{Demographics}

First, we visually checked the quality of the CBF map, and the 3D high-resolution brain structural images for all the patients using the methods on reference (23). Skull distortions in TBI patients can make difficult to normalize neuroimaging data. After carefully visually inspecting the brain images, we excluded 13 patients from the recruited patients for head motion, brain deformation, artifact, or severe hydrocephalus.

Fifteen patients with VS ranged in age from 23 to 71 years, and included 5 women and 10 men. Eight patients met the MCS criteria, and they ranged in age from 23 to 61 years, and included one woman and seven men. Etiologies included traumatic brain injury, stroke, and hypoxic-ischemic encephalopathy (Table 1). Patients were within an interval from injury to evaluation ranging from 1 month to 47 months. Twenty-seven healthy volunteers (13 women and 14 men) were identified, ranging in age from 28 to 56 years. The differences of etiology, age, sex, and duration of disease were not statistically significant.

\section{CBF Differences: Patients vs. Control Subjects}

The 10 typical ROIs CBF of the control subjects ranged from 15.3 to 98.3 (median value 54.5 ) $\mathrm{mL} / 100 \mathrm{~g} / \mathrm{min}$ on PLD $1.5 \mathrm{~s}$ and from 22.4 to $86.5 \mathrm{~mL} / 100 \mathrm{~g} / \mathrm{min}$ (median value 30.2 ) on PLD
$2.5 \mathrm{~s}$ (Figure 1). The 10 typical ROIs CBF of the MCS and VS patients showed greater variability and ranged from 4.1 to 83.3 (median value 54.2 ) $\mathrm{mL} / 100 \mathrm{~g} / \mathrm{min}$ on PLD $1.5 \mathrm{~s}$ and from 7.8 to 60.9 (median value 39.8 ) $\mathrm{mL} / 100 \mathrm{~g} / \mathrm{min}$ on PLD $2.5 \mathrm{~s}$.

\section{PLD 1.5 vs. $2.5 \mathrm{~s}$}

The mean CBF for control subjects and patients on PLD 1.5 and $2.5 \mathrm{~s}$ was calculated for each of the 10 ROIs. For most regions in the control subjects, mean CBF changed less than did PLD from 1.5 to $2.5 \mathrm{~s}$ (Figure 1). On the contrary, mean CBF increased for most ROIs of patients as PLD changed from 1.5 to $2.5 \mathrm{~s}$. In general, the difference of mean CBF between MCS patients and control subjects was smaller on PLD $2.5 \mathrm{~s}$ than on $1.5 \mathrm{~s}$.

\section{Ten Typical ROIs}

The mean CBF for control subjects was significantly higher than that for patients for nine ROIs on PLD $1.5 \mathrm{~s}$, except the occipital pole. CBF for control subjects was significantly higher than that for patients for all 10 ROIs on PLD $2.5 \mathrm{~s}$.

\section{AAL-116 Template}

The mean CBF for control subjects was significantly higher than that for patients for every ROI on PLD $1.5 \mathrm{~s}$, whereas the mean CBF for control subjects was significantly higher than that for patients for every ROI on PLD $2.5 \mathrm{~s}$ except bilateral pallidum (PAL).

\section{Bilateral Brodmann's Template}

The mean CBF for control subjects was significantly higher than that for patients for every ROI on PLD $1.5 \mathrm{~s}$ except the lateral globus pallidus, medial globus pallidus, and hypothalamus, whereas the mean $\mathrm{CBF}$ for control subjects was significantly

TABLE 1 | Patient data.

\begin{tabular}{|c|c|c|c|c|c|}
\hline Case no. & Diagnosis according to CRS-R & CRS-R total score (and subscores ${ }^{a}$ ) & Etiology & Lesions $^{\mathrm{b}}$ on T1WI L/R/B/N & Duration (months) \\
\hline 1 & MCS & $17(3,4,5,1,1,3)$ & $\mathrm{T}$ & $\mathrm{N}$ & 1 \\
\hline 2 & MCS & $10(2,3,2,1,0,2)$ & n-T (anoxia) & $\mathrm{B}$ & 3 \\
\hline 3 & MCS & $9(1,3,2,1,0,2)$ & $\mathrm{T}$ & $\mathrm{N}$ & 6 \\
\hline 4 & MCS & $11(1,3,4,1,0,2)$ & $\mathrm{T}$ & $\mathrm{L}$ & 2 \\
\hline 5 & MCS & $7(1,0,3,1,0,2)$ & $\mathrm{T}$ & $L$ & 3 \\
\hline 6 & MCS & $11(3,3,2,1,0,2)$ & $\mathrm{T}$ & $\mathrm{N}$ & 7 \\
\hline 7 & MCS & $9(1,3,2,1,0,2)$ & $\mathrm{T}$ & $\mathrm{R}$ & 2 \\
\hline 8 & MCS & $8(1,1,3,1,0,2)$ & $\mathrm{T}$ & $\mathrm{R}$ & 47 \\
\hline 9 & VS & $7(1,1,2,1,0,2)$ & $\mathrm{T}$ & $\mathrm{L}$ & 1 \\
\hline 10 & VS & $7(1,1,2,1,0,2)$ & n-T (stroke) & $L$ & 1 \\
\hline 11 & VS & $5(0,0,2,1,0,2)$ & n-T (anoxia) & $\mathrm{B}$ & 9 \\
\hline 12 & VS & $6(1,0,2,1,0,2)$ & n-T (anoxia) & $\mathrm{B}$ & 4 \\
\hline 13 & VS & $7(1,1,2,1,0,2)$ & n-T (stroke) & $\mathrm{R}$ & 6 \\
\hline 14 & VS & $7(1,1,2,1,0,2)$ & n-T (anoxia) & $\mathrm{B}$ & 2 \\
\hline 15 & VS & $3(1,0,1,1,0,0)$ & $\mathrm{T}$ & $\mathrm{R}$ & 6 \\
\hline 16 & VS & $5(1,1,2,1,0,0)$ & $\mathrm{T}$ & $\mathrm{L}$ & 1 \\
\hline 17 & VS & $7(1,1,2,1,0,2)$ & $\mathrm{T}$ & $\mathrm{L}$ & 3 \\
\hline 18 & VS & $5(0,0,2,1,0,2)$ & n-T (anoxia) & $\mathrm{B}$ & 2 \\
\hline 19 & VS & $6(1,0,2,1,0,2)$ & n-T (anoxia) & B & 8 \\
\hline 20 & VS & $6(1,0,1,2,0,2)$ & $\mathrm{T}$ & $R$ & 4 \\
\hline 21 & VS & $6(1,1,2,1,0,1)$ & $\mathrm{T}$ & $\mathrm{R}$ & 3 \\
\hline 22 & VS & $6(1,0,2,1,0,2)$ & $\mathrm{T}$ & $\mathrm{R}$ & 17 \\
\hline 23 & VS & $6(1,1,1,1,0,2)$ & $\mathrm{T}$ & $\mathrm{L}$ & 3 \\
\hline
\end{tabular}

$T$, traumatic; $n-T$, non-traumatic.

${ }^{a}$ Coma Recovery Score-Revised (CRS-R) subscores are in the following order: auditory, visual, motor, verbal, communication, and arousal.

'Lesions on T1WI: the L, R, B, and N equal left, right, bilateral, and none lesions on the cerebral hemispheres. 

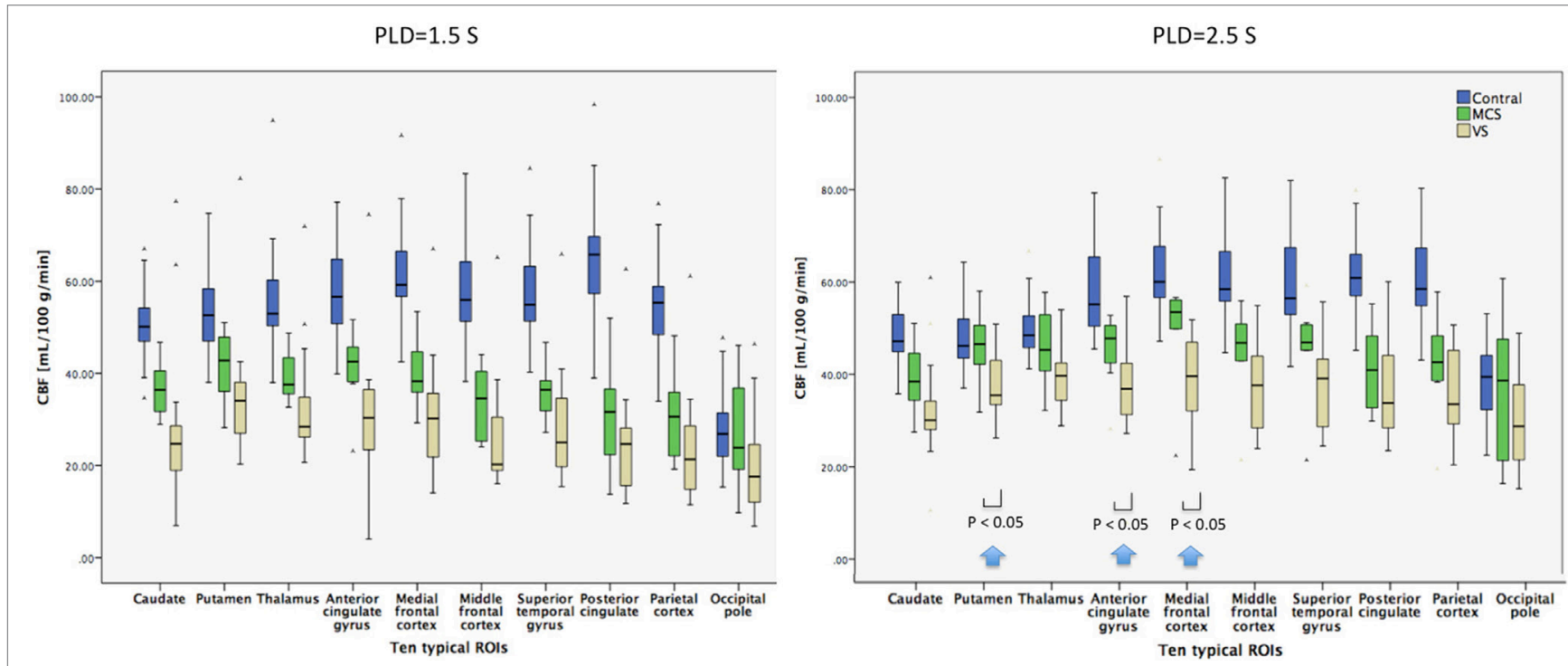

FIGURE 1 | Box plots displaying 25, median value, 75 (box), and 95\% (whiskers) of cerebral blood flow (CBF) value distributions in the 10 typical regions of interest (ROIs) of healthy control subjects, minimally conscious state (MCS) patients, and vegetative state (VS) patients. Compared with VS patients, the regional mean CBF with post-labeled delay (PLD) $1.5 \mathrm{~s}$ for MCS patients had a pattern of relatively decreased CBF in most ROls, but not significantly. As the PLD changed to $2.5 \mathrm{~s}$, the regional mean CBF for MCS had a pattern of significantly increased CBF in the regions including the putamen, anterior cingulate gyrus, and medial frontal cortex $(P<0.05)$ (as shown by arrows).

higher than that for patients for every ROI on PLD $2.5 \mathrm{~s}$ except Brodmann's area 27, the hypothalamus, medial geniculum body, PUT, ventral lateral nucleus, lateral posterior nucleus, lateral globus pallidus, ventral posterior medial nucleus, medial globus pallidus, ventral posterior lateral nucleus, lateral geniculum body, and subthalamic nucleus.

\section{GM and WM}

The mean CBF of GM and WM for control subjects was significantly higher than that for patients on PLD 1.5 and $2.5 \mathrm{~s}$.

\section{CBF Differences: MCS vs. VS}

\section{Ten Typical ROls}

Compared with VS patients, the regional mean CBF with PLD $1.5 \mathrm{~s}$ for MCS had a pattern of relatively decreased CBF in most ROIs, but not significantly. As the PLD changed to $2.5 \mathrm{~s}$; however, the regional mean $\mathrm{CBF}$ for MCS had a pattern of significantly increased $\mathrm{CBF}$ in the regions including PUT, anterior cingulate gyrus, and medial frontal cortex $(P=0.0164,0.0497$, and 0.0288) (Figure 1).

\section{AAL-116 Template}

Compared with VS patients, the regional CBF with PLD $1.5 \mathrm{~s}$ for MCS had a pattern of relatively increased CBF in the regions of left inferior frontal gyrus, opercular (F3OP), precentral gyrus (PRE), rolandic operculum (RO), insula (IN), PUT, PAL, and bilateral gyrus rectus $(\mathrm{GR})(P<0.05)$. The regional $\mathrm{CBF}$ with PLD $2.5 \mathrm{~s}$ for MCS had a pattern of relatively increased CBF in the regions including left inferior F3OP, inferior frontal gyrus, triangular (F3T), PRE, RO, temporal pole: middle temporal gyrus (T2P), temporal pole: superior temporal gyrus (T1P), IN, PUT, THAL, and right GR $(P<0.05)$ (Figure 2; Table 2).

\section{Bilateral Brodmann's Template}

Compared with VS patients, the regional CBF with PLD $1.5 \mathrm{~s}$ for MCS had a pattern of relatively increased CBF in the regions of bilateral medial globus pallidus, Brodmann's area 24, Brodmann's area 44 , hypothalamus, and optic tract $(P<0.05)$. The regional CBF with PLD $2.5 \mathrm{~s}$ for MCS had a pattern of relatively increased $\mathrm{CBF}$ in the regions including Brodmann's area 9, Brodmann's area 13 , Brodmann's area 24, Brodmann's area 28, Brodmann's area 32, Brodmann's area 33, Brodmann's area 34, Brodmann's area 38, Brodmann's area 44, medial dorsal nucleus, ventral lateral nucleus, PUT, hypothalamus, and caudate head $(P<0.05)$ (Figure 3 ).

\section{GM and WM}

The mean CBF of GM or WM for MCS was not significantly higher than that for VS on PLD 1.5 or $2.5 \mathrm{~s}$ (Figure 4).

\section{DISCUSSION}

Our study demonstrates globally reduced ASL-measured CBF in MCS or VS patents compared with normal controls, particularly with PLD $1.5 \mathrm{~s}$. Compared with VS, the regional CBF for MCS had a pattern of significantly increased CBF in the regions including the PUT, anterior cingulate gyrus, and medial frontal cortex with PLD $2.5 \mathrm{~s}$, within 10 typical ROIs., In addition, a left-lateralized pattern is observed to differentiate MCS from VS on AAL-116 template. Moreover, regional CBF with PLD 2.5 s could find more regions of pattern differentiating MCS from VS than with PLD $1.5 \mathrm{~s}$, except for globus pallidus.

This study found globally reduced ASL-measured CBF in MCS or VS patients compared with normal controls, particularly with 


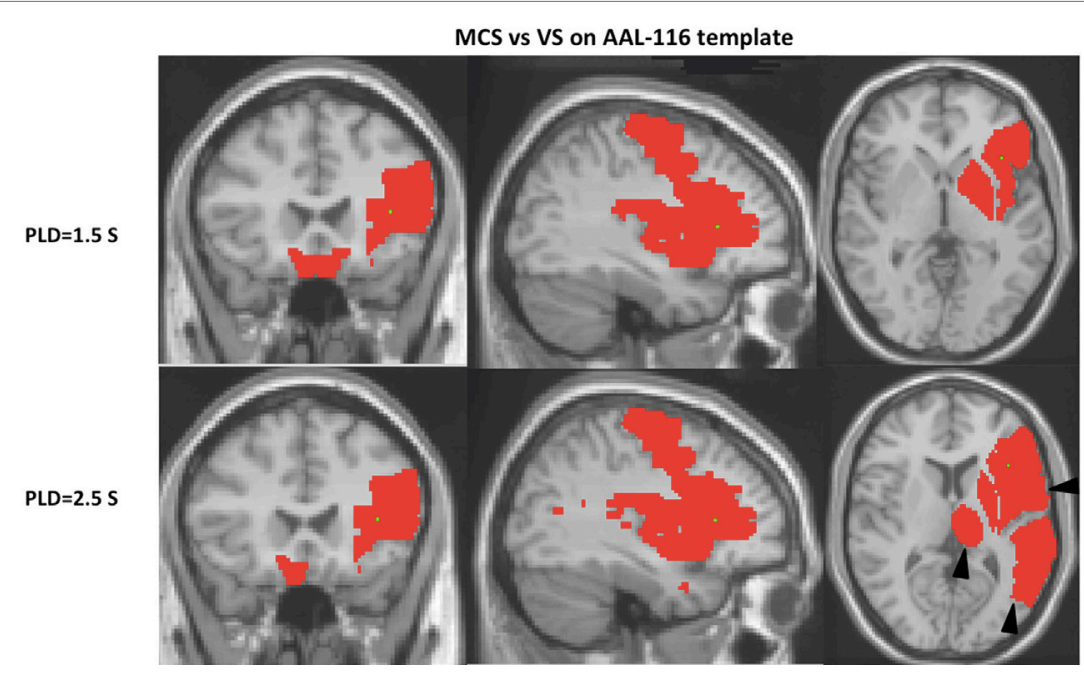

FIGURE 2 | On the Automated Anatomical Labeling-116 (AAL-116) template, a left-lateralized pattern is observed. With regard to the cerebral blood flow (CBF) of single AAL regions separating vegetative state (VS) from minimally conscious state (MCS), the MCS regions were generally left-lateralized. CBF with post-labeled delay (PLD) $2.5 \mathrm{~s}$ found more regions of pattern differentiating MCS from VS than with PLD $1.5 \mathrm{~s}$ on AAL-116 templates, including the inferior left frontal gyrus, triangular (F3T), temporal pole: middle temporal gyrus (T2P), temporal pole: superior temporal gyrus (T1P), and thalamus (THAL) (as shown by triangles)

TABLE 2 | The $P$-values of regional region-of-interest (ROI) cerebral blood flow between minimally conscious state and vegetative state patients on the Automated Anatomical Labeling-116 template with two post-labeled delay (PLD).

\begin{tabular}{llll}
\hline PLD & L/R & ROI name & P-value \\
\hline $1.5 \mathrm{~s}$ & $\mathrm{~L}$ & Inferior frontal gyrus, opercular (F3OP) & 0.015 \\
$1.5 \mathrm{~s}$ & $\mathrm{~L}$ & Precentral gyrus (PRE) & 0.047 \\
$1.5 \mathrm{~s}$ & $\mathrm{~L}$ & Rolandic operculum (RO) & 0.034 \\
$1.5 \mathrm{~s}$ & $\mathrm{~L}$ & Insula (IN) & 0.034 \\
$1.5 \mathrm{~s}$ & $\mathrm{~L}$ & Putamen (PUT) & 0.049 \\
$1.5 \mathrm{~s}$ & $\mathrm{~L}$ & Pallidum (PAL) & 0.037 \\
$1.5 \mathrm{~s}$ & $\mathrm{~L}$ & Gyrus rectus (GR) & 0.049 \\
$1.5 \mathrm{~s}$ & $\mathrm{R}$ & Gyrus rectus (GR) & 0.048 \\
$2.5 \mathrm{~s}$ & $\mathrm{~L}$ & Inferior frontal gyrus, opercular (F3OP) & 0.028 \\
$2.5 \mathrm{~s}$ & $\mathrm{~L}$ & Inferior frontal gyrus, triangular (F3T) & 0.047 \\
$2.5 \mathrm{~s}$ & $\mathrm{~L}$ & Precentral gyrus (PRE) & 0.041 \\
$2.5 \mathrm{~s}$ & $\mathrm{~L}$ & Rolandic operculum (RO) & 0.030 \\
$2.5 \mathrm{~s}$ & $\mathrm{~L}$ & Temporal pole: middle temporal gyrus (T2P) & 0.014 \\
$2.5 \mathrm{~s}$ & $\mathrm{~L}$ & Temporal pole: superior temporal gyrus (T1P) & 0.006 \\
$2.5 \mathrm{~s}$ & $\mathrm{~L}$ & Insula (IN) & 0.018 \\
$2.5 \mathrm{~s}$ & $\mathrm{~L}$ & Putamen (PUT) & 0.039 \\
$2.5 \mathrm{~s}$ & $\mathrm{~L}$ & Thalamus (THAL) & 0.049 \\
$2.5 \mathrm{~s}$ & $\mathrm{R}$ & Gyrus rectus (GR) & 0.045 \\
\hline
\end{tabular}

PLD $1.5 \mathrm{~s}$, which were similar with previous studies with ASL (12) and PET (24). Our finding of decreased relative blood flow to the medial frontal cortex and frontal areas in our MCS or VS patients is consistent with previous work highlighting the vulnerability of the anterior forebrain after severe brain injury. Various brain injuries ultimately disrupt the corticostriatopallidal-thalamocortical projection system's ability to modulate the anterior forebrain (25). In this study, the CBF of the area of the PUT, PAL, and THAL was higher in MSC than in VS.

Preserved relative blood flow to the medial frontal cortex, left temporal-parietal areas, and left THAL, which were parts of the default mode network (DMN), appears to be another defining characteristic of our sample of MCS patients, consistent with emerging functional MRI (fMRI) research. The DMN consists of a set of regions, including the medial prefrontal cortex, precuneous/ posterior cingulate, bilateral temporal-parietal areas, and THAL, which are more active at rest than during attention-demanding tasks (26). The DMN has been proposed as the substrate for consciousness (27). It was also reported that fMRI connectivity to the precuneous/posterior cingulate region could differentiate MCS patients from unconscious patients (28). It remains to be investigated whether MCS and VS can be differentiated by different patterns of regional CBF as measured by ASL.

On the AAL-116 template, as we choose the ROIs left and right separately, a left-lateralized pattern is observed. With regard to the $\mathrm{CBF}$ of single AAL regions, separating VS from MCS, the MCS regions were generally left-lateralized. Rosazza et al. found that the differences between VS and MCS patients were stronger for the left than the right hemisphere for resting-state fMRI and 18F-fluorodeoxyglucose (FDG)-PET, and that the integrity of the left hemisphere is predictive of better clinical status (29). Based on behavioral observation of patients, it has also been hypothesized that the functional preservation of the left hemispheric function has greater impact on level of consciousness than the right one (e.g., Serafetinides et al. (30), Glosser et al. (31), and Meador et al. (32)). The study based on VBM found that GM atrophy in disorders of consciousness appeared to be mostly left lateralized (33). Left-lateralized atrophy in disorders of consciousness also made a tentative association with severely impaired language processing $(34,35)$. Cerebral atrophy has been shown to be associated with globally decreased blood flow $(36,37)$. However, as the evidence for left lateralization of the neural foundations of consciousness remains controversial, further confirmation of this finding is required. 


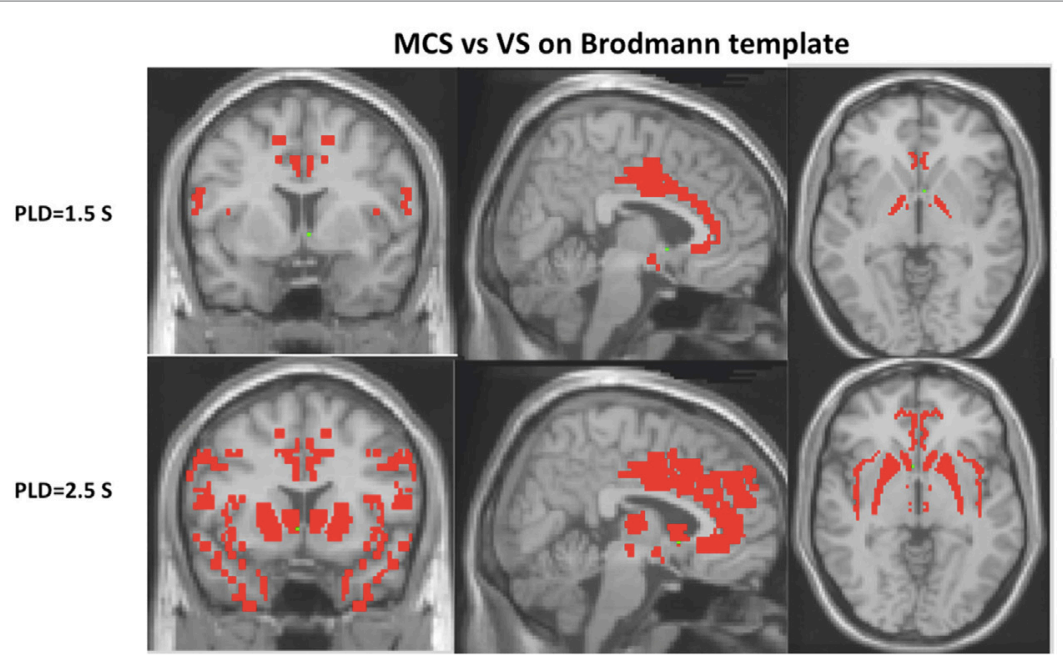

FIGURE 3 | Cerebral blood flow with post-labeled delay (PLD) 2.5 s could find more regions of pattern differentiating minimally conscious state (MCS) from vegetative state (VS) than with PLD 1.5 s on Brodmann's template, including Brodmann's areas 9, 13, 28, 32, 33, 34, 38, medial dorsal nucleus, ventral lateral nucleus, putamen, and caudate head $(P<0.05)$.

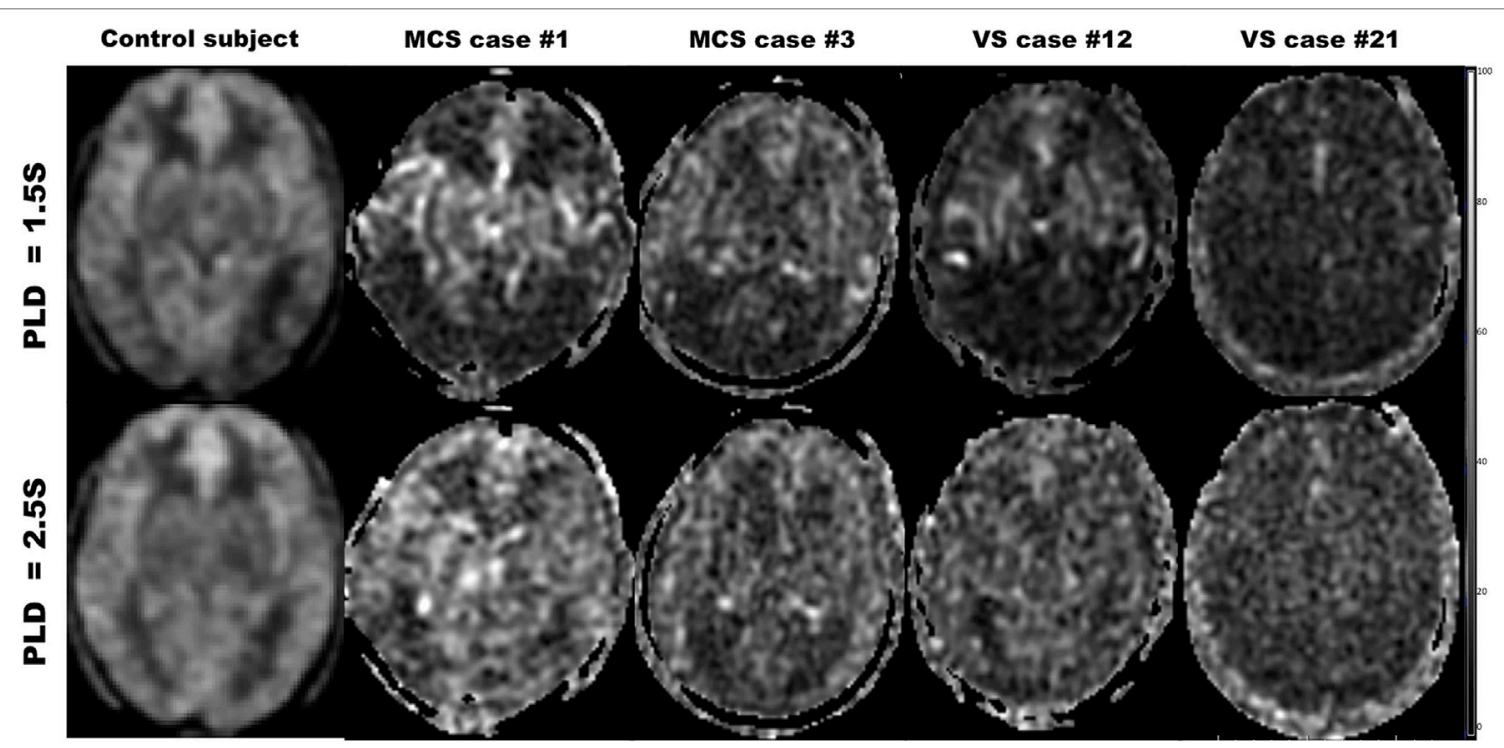

FIGURE 4 | Cerebral blood flow maps with post-labeled delay (PLD) 1.5 and $2.5 \mathrm{~s}$ for the healthy control subject, minimally conscious state (MCS) patients, and vegetative state (VS) patients.

Different PLDs of pcASL had considerable influence on MCS and VS. CBF with PLD $2.5 \mathrm{~s}$ could find more regions of pattern differentiating MCS from VS than with PLD $1.5 \mathrm{~s}$ on the AAL116 or Brodmann's template, including inferior left F3T, T2P, T1P, THAL, Brodmann's areas 9, 13, 28, 32, 33, 34, 38, medial dorsal nucleus, ventral lateral nucleus, PUT, and caudate head. On one hand, longer PLD means more blood flows into the regional brain $(16,38,39)$. On the other hand, longer PLD will decrease the SNR of ASL, as the magnetically labeled water in ASL only has 1-3 s of half-life. Moreover, the spins are exposed to the presence of a substantial amount of iron in the basal ganglia regions, resulting in further T1 shortening (40) and lower apparent $\mathrm{CBF}$ values with longer PLD. The apparent regional ASL CBF was the result of the balance among the aforementioned three aspects at least (16). For MCS and VS patients, longer PLD made the regional CBF higher than short PLD, especially for MCS. Therefore, CBF with PLD $2.5 \mathrm{~s}$ could find more regions of pattern differentiating MCS from VS than with PLD $1.5 \mathrm{~s}$ (Figure 4). However, as for globus pallidus, the substantial amount of iron could make the apparent CBF lower on PLD 2.5 s. Thus, only CBF with PLD 1.5 s could find the difference of globus pallidus CBF between MSC and VS (Figure 4). 
One of the major limitations in our study was motion artifact, a common problem in ASL. Although our methods selected for data not significantly motion-degraded, even slight motion artifact disturbed their CBF values. Another consideration in interpreting our results is the inter-subject variation, such as age and gender. Previous work has demonstrated that older subjects have significantly decreased CBF compared with younger counterparts, especially to the frontal cortex $(36,41)$. Likewise, women overall have increased global CBF, approximately $13 \%$ higher than men (36). Moreover, our patients were studied in 1 to 47 months after brain injury, possibly introducing serious bias in the patient selection. There is some consensus that the late subacute phase (days 14-20) may be optimal for imaging. By this time, brain edema has subsided, and many critical decisions in medical and ethical management have been made (42). However, the short time (i.e., 1-2 months) could imply uncertainty or fluctuation of clinical diagnosis, since these patients at this time could have high probability of ongoing clinical evolution. More investigation on the use of functional imaging in general, including ASL, is needed at this pivotal stage in medical decision-making. Finally, the sample size was very different among patients in an MCS $(n=8)$, patients with VS $(n=15)$, and healthy control subjects $(n=23)$. The different sample sizes between MCS and VS patients might explain the lack of statistical significance in some results.

\section{CONCLUSION}

We identified a selective reduction of CBF within specific brain regions in VS patients compared with MCS patients. Therefore, MCS might be differentiated from VS by different ranges of regional $\mathrm{CBF}$ as measured by ASL. Multi-PLD ASL may serve as an adjunctive method to separate MCS from VS and assess functional reserve in patients recovering from severe brain injuries.

\section{REFERENCES}

1. Giacino JT, Fins JJ, Laureys S, Schiff ND. Disorders of consciousness after acquired brain injury: the state of the science. Nat Rev Neurol (2014) 10:99-114. doi:10.1038/nrneurol.2013.279

2. Schnakers C, Vanhaudenhuyse A, Giacino J, Ventura M, Boly M, Majerus S, et al. Diagnostic accuracy of the vegetative and minimally conscious state: clinical consensus versus standardized neurobehavioral assessment. BMC Neurol (2009) 9:35. doi:10.1186/1471-2377-9-35

3. Wang DJ, Alger JR, Qiao JX, Hao Q, Hou S, Fiaz R, et al. The value of arterial spin-labeled perfusion imaging in acute ischemic stroke: comparison with dynamic susceptibility contrast-enhanced MRI. Stroke (2012) 43:1018-24. doi:10.1161/STROKEAHA.111.631929

4. Binnewijzend MA, Kuijer JP, Benedictus MR, van der Flier WM, Wink AM, Wattjes MP, et al. Cerebral blood flow measured with 3D pseudocontinuous arterial spin-labeling MR imaging in Alzheimer disease and mild cognitive impairment: a marker for disease severity. Radiology (2013) 267:221-30. doi:10.1148/radiol.12120928

5. Järnum H, Steffensen EG, Knutsson L, Fründ ET, Simonsen CW, LundbyeChristensen S, et al. Perfusion MRI of brain tumours: a comparative study of pseudo-continuous arterial spin labelling and dynamic susceptibility contrast imaging. Neuroradiology (2010) 52:307-17. doi:10.1007/s00234-009-0616-6

6. Detre JA, Rao H, Wang DJ, Chen YF, Wang Z. Applications of arterial spin labeled MRI in the brain. J Magn Reson Imaging (2012) 35:1026-37. doi:10.1002/jmri.23581

7. Lyu J, Ma N, Liebeskind DS, Wang DJ, Ma L, Xu Y, et al. Arterial spin labeling magnetic resonance imaging estimation of antegrade and collateral
Because of its advantages of speed and ease of acquisition and its ability to provide precise quantitative CBF, ASL could be used in longitudinal assessments of patients with severe brain injuries.

\section{ETHICS STATEMENT}

All procedures performed in the studies involving human participants were in accordance with the ethical standards of the institutional review boards of PLA Army General Hospital and with the 1964 Helsinki Declaration and its later amendments or comparable ethical standards. The datasets generated during and/or analyzed during the current study are available from the corresponding author on reasonable request.

\section{AUTHOR NOTE}

Statistical Analysis Conducted by Dr. Bing Wu, MD, PLA Army General Hospital.

\section{AUTHOR CONTRIBUTIONS}

BW and YY contributed to the study concept and design, and analysis and interpretation of data. SZ, WW, ZW, and GH contributed to the acquisition, analysis, and interpretation of data. $\mathrm{JH}$ and XW contributed to the study concept and design, and critical revision of the manuscript.

\section{ACKNOWLEDGMENTS}

The authors thank Ms. Mingmei Ge, Ms. Xiangfeng Meng from PLA Army General Hospital, and Mr. Hai Song from GE Healthcare for providing technical support.

flow in unilateral middle cerebral artery stenosis. Stroke (2016) 47:428-33. doi:10.1161/STROKEAHA.115.011057

8. Wang Y, West JD, Bailey JN, Westfall DR, Xiao H, Arnold TW, et al. Decreased cerebral blood flow in chronic pediatric mild TBI: an MRI perfusion study. Dev Neuropsychol (2015) 40:40-4. doi:10.1080/87565641.2014.979927

9. Ge Y, Patel MB, Chen Q, Grossman EJ, Zhang K, Miles L, et al. Assessment of thalamic perfusion in patients with mild traumatic brain injury by true FISP arterial spin labelling MR imaging at 3T. Brain Inj (2009) 23:666-74. doi:10.1080/02699050903014899

10. Buxton RB, Frank LR. A model for the coupling between cerebral blood flow and oxygen metabolism during neural stimulation. J Cereb Blood Flow Metab (1997) 17:64-72. doi:10.1097/00004647-199701000-00009

11. Laureys S, Owen AM, Schiff ND. Brain function in coma, vegetative state, and related disorders. Lancet Neurol (2004) 3:537-46. doi:10.1016/S1474-4422 (04)00852-X

12. Liu AA, Voss HU, Dyke JP, Heier LA, Schiff ND. Arterial spin labeling and altered cerebral blood flow patterns in the minimally conscious state Neurology (2011) 77:1518-23. doi:10.1212/WNL.0b013e318233b229

13. Giacino JT, Ashwal S, Childs N, Cranford R, Jennett B, Katz DI, et al. The minimally conscious state: definition and diagnostic criteria. Neurology (2002) 58:349-53. doi:10.1212/WNL.58.3.349

14. Giacino JT. The minimally conscious state: defining the borders of consciousness. Prog Brain Res (2005) 150:381-95. doi:10.1016/S0079-6123(05) 50027-X

15. Dai W, Garcia D, de Bazelaire C, Alsop DC. Continuous flow-driven inversion for arterial spin labeling using pulsed radio frequency and gradient fields. Magn Reson Med (2008) 60:1488-97. doi:10.1002/mrm.21790 
16. Wu B, Lou X, Wu X, Ma L. Intra- and interscanner reliability and reproducibility of 3D whole-brain pseudo-continuous arterial spin-labeling MR perfusion at 3T. J Magn Reson Imaging (2014) 409:402-9. doi:10.1002/jmri.24175

17. Ma Y, Huang C, Dyke JP, Pan H, Alsop D, Feigin A, et al. Parkinson's disease spatial covariance pattern: noninvasive quantification with perfusion MRI. J Cereb Blood Flow Metab (2010) 30:505-9. doi:10.1038/jcbfm.2009.256

18. Xu G, Rowley HA, Wu G, Alsop DC, Shankaranarayanan A, Dowling M, et al. Reliability and precision of pseudo-continuous arterial spin labeling perfusion MRI on 3.0 T and comparison with 15O-water PET in elderly subjects at risk for Alzheimer's disease. NMR Biomed (2010) 23:286-93. doi:10.1002/ nbm. 1462

19. Lee C, Lopez OL, Becker JT, Raji C, Dai W, Kuller LH, et al. Imaging cerebral blood flow in the cognitively normal aging brain with arterial spin labeling: implications for imaging of neurodegenerative disease. J Neuroimaging (2009) 19:344-52. doi:10.1111/j.1552-6569.2008.00277.x

20. Tzourio-Mazoyer N, Landeau B, Papathanassiou D, Crivello F, Etard O, Delcroix N, et al. Automated anatomical labeling of activations in SPM using a macroscopic anatomical parcellation of the MNI MRI single-subject brain. Neuroimage (2002) 15:273-89. doi:10.1006/nimg.2001.0978

21. Alsop DC, Casement M, de Bazelaire C, Fong T, Press DZ. Hippocampal hyperperfusion in Alzheimer's disease. Neuroimage (2008) 42:1267-74. doi:10.1016/j.neuroimage.2008.06.006

22. van Gelderen P, de Zwart JA, Duyn JH. Pittfalls of MRI measurement of white matter perfusion based on arterial spin labeling. Magn Reson Med (2008) 59:788-95. doi:10.1002/mrm.21515

23. Weng L, Xie Q, Zhao L, Zhang R, Ma Q, Wang J, et al. Abnormal structural connectivity between the basal ganglia, thalamus, and frontal cortex in patients with disorders of consciousness. Cortex (2017) 90:71-87. doi:10.1016/j. cortex.2017.02.011

24. Levy DE, Sidtis JJ, Rottenberg DA, Jarden JO, Strother SC, Dhawan V, et al. Differences in cerebral blood flow and glucose utilization in vegetative versus locked-in patients. Ann Neurol (1987) 22:673-82. doi:10.1002/ana.410220602

25. Fridman EA, Beattie BJ, Broft A, Laureys S, Schiff ND. Regional cerebral metabolic patterns demonstrate the role of anterior forebrain mesocircuit dysfunction in the severely injured brain. Proc Natl Acad Sci U S A (2014) 111:6473-8. doi:10.1073/pnas.1320969111

26. Raichle ME, MacLeod AM, Snyder AZ, Powers WJ, Gusnard DA, Shulman GL. A default mode of brain function. Proc Natl Acad Sci U S A (2001) 98:676-82. doi:10.1073/pnas.98.2.676

27. Boly M, Phillips C, Tshibanda L, Vanhaudenhuyse A, Schabus M, Dang-Vu TT, et al. Intrinsic brain activity in altered states of consciousness: how conscious is the default mode of brain function? Ann N Y Acad Sci (2008) 1129:119-29. doi:10.1196/annals.1417.015

28. Vanhaudenhuyse A, Noirhomme Q, Tshibanda LJ, Bruno MA, Boveroux P, Schnakers C, et al. Default network connectivity reflects the level of consciousness in non-communicative brain-damaged patients. Brain (2010) 133:161-71. doi:10.1093/brain/awp313

29. Rosazza C, Andronache A, Sattin D, Bruzzone MG, Marotta G, Nigri A, et al. Multimodal study of default-mode network integrity in disorders of consciousness. Ann Neurol (2016) 79:841-53. doi:10.1002/ana.24634

30. Serafetinides EA, Hoare RD, Driver M. Intracarotid sodium amylobarbitone and cerebral dominance for speech and consciousness. Brain (1965) 88:107-30. doi:10.1093/brain/88.1.107
31. Glosser G, Cole LC, Deutsch GK, Donofrio N, Bagley L, Baltuch G, et al. Hemispheric asymmetries in arousal affect outcome of the intracarotid amobarbital test. Neurology (1999) 52:1583-90. doi:10.1212/WNL.52.8.1583

32. Meador KJ, Loring DW, Lee GP, Nichols ME, Moore EE, Figueroa RE. Level of consciousness and memory during the intracarotid sodium amobarbital procedure. Brain Cogn (1997) 33:178-88. doi:10.1006/brcg.1997.0891

33. Guldenmund P, Soddu A, Baquero K, Vanhaudenhuyse A, Bruno MA, Gosseries O, et al. Structural brain injury in patients with disorders of consciousness: a voxel-based morphometry study. Brain Inj (2016) 30:343-52. doi:10.3109/02699052.2015.1118765

34. Weiss N, Galanaud D, Carpentier A, Tezenas de Montcel S, Naccache L, Coriat $\mathrm{P}$, et al. A combined clinical and MRI approach for outcome assessment of traumatic head injured comatose patients. J Neurol (2008) 255:217-23. doi:10.1007/s00415-008-0658-4

35. Scott SK. The neurobiology of speech perception and production - can functional imaging tell us anything we did not already know? J Commun Disord (2012) 45:419-25. doi:10.1016/j.jcomdis.2012.06.007

36. Parkes LM, Rashid W, Chard DT, Tofts PS. Normal cerebral perfusion measurements using arterial spin labeling: reproducibility, stability, and age and gender effects. Magn Reson Med (2004) 51:736-43. doi:10.1002/mrm.20023

37. Deibler AR, Pollock JM, Kraft RA, Tan H, Burdette JH, Maldjian JA. Arterial spin-labeling in routine clinical practice, part 2: hypoperfusion patterns. AJNR Am J Neuroradiol (2008) 29:1235-41. doi:10.3174/ajnr.A1033

38. Fan AP, Guo J, Khalighi MM, Gulaka PK, Shen B, Park JH, et al. Long-delay arterial spin labeling provides more accurate cerebral blood flow measurements in moyamoya patients: a simultaneous positron emission tomography/ MRI study. Stroke (2017) 48:2441-9. doi:10.1161/STROKEAHA.117. 017773

39. Fan AP, Jahanian H, Holdsworth SJ, Zaharchuk G. Comparison of cerebral blood flow measurement with [15O]-water positron emission tomography and arterial spin labeling magnetic resonance imaging: a systematic review. J Cereb Blood Flow Metab (2016) 36:842-61. doi:10.1177/0271678X16636393

40. Pfefferbaum A, Adalsteinsson E, Rohlfing T, Sullivan EV. Diffusion tensor imaging of deep gray matter brain structures: effects of age and iron concentration. Neurobiol Aging (2010) 31:482-93. doi:10.1016/j.neurobiolaging. 2008.04.013

41. Dupui P, Guell A, Bessoles G, et al. Cerebral blood flow in aging. Decrease of hyperfrontal distribution. Monogr Neural Sci (1984) 11:131-8.

42. Tshibanda L, Vanhaudenhuyse A, Boly M, Soddu A, Bruno MA, Moonen G, et al. Neuroimaging after coma. Neuroradiology (2010) 52:15-24. doi:10.1007/ s00234-009-0614-8

Conflict of Interest Statement: The authors declare that the research was conducted in the absence of any commercial or financial relationships that could be construed as a potential conflict of interest.

Copyright (c) $2018 \mathrm{Wu}$, Yang, Zhou, Wang, Wang, Hu, He and Wu. This is an openaccess article distributed under the terms of the Creative Commons Attribution License (CC BY). The use, distribution or reproduction in other forums is permitted, provided the original author(s) and the copyright owner are credited and that the original publication in this journal is cited, in accordance with accepted academic practice. No use, distribution or reproduction is permitted which does not comply with these terms. 